\title{
k-18. The Attitude of Arterio-venous System in the Brain and Spinal Cord studied by the Use of Specimens with India Ink Injection in Experimental Whiplash Injury
}

\author{
Yukio Mivra, Hiroshi Hiranuma and Takashi Tanaka \\ Department of Orthopaedic Surgery, Tokyo Medical College \\ Gundai KaWAHARA \\ Department of Anatomy, Tokyo Medical College
}

In an attempt to elucidate the pathologic physiology of whiplash injury, various degrees of whiplash injury was produced in mature rabbits by swinging shock apparatus followed by whole blood letting. An India ink was injected into the arterial-venous system with the method developed by us. After fixing the head and neck en bloc, slice sections were prepared through the sagittal, frontal and transverse planes to study the attitude of the arterial-venous system in the brain, cervical cord and cervical spinal nerve.

Experimental results were variable according to the degree of shock. In the group given an intense shock, the arterial system failed to show a remarkable change in the brain, but arteriolar diapedesis or thexis was found in the gray matter of the superior cervical cord and brain stem. In the venous system, congestion of venous blood in the capillary range and venular hemorrhage was noted.

We have therefore emphasized the circulatory disturbance in the arterioles, venules and capillaries.

\section{k-19. Clinico-pathological Study of Whiplash Injury}

\author{
Masumi Yoshioka, Norio Yoshimasu and Akira Teraoka \\ Department of Neurosurgery, The First National Hospital of Tokyo
}

Pathophysiological basis of clinical syndrome of whiplash injury is still obscure. In order to approach the pathophysiological basis of its syndrome, air myelography was performed in the cases of whiplash injury, which have headache, beaviness of head, dizziness, tinnitus, blurred vision, syncope attack, neck pain, back pain, stiffness of shoulder, ataxia, sensory and motor disturbances of extremities etc.

Air myelogram showed non-filling of the air anterior and/or posterior to the cervical spinal cord in the subarachnoid space. This finding may reveal a relative narrowing of the subarachnoid space, which can cause a compression of 
the spinal cord.

Under the finding, laminectomy was performed in two cases $\left(\mathrm{C}_{2}-\mathrm{C}_{7}, \mathrm{C}_{1}-\mathrm{Th}_{2}\right)$. After operation, some clinical symptoms disappeared or reduced. Heaviness of head, dizziness, blurred vision, syncope attack disappeared and sensory-motor disturbances, ataxia, headache, tinnitus reduced. Stiffness of neck and shoulder remained.

Postoperative air myelogram showed filling of the air anterior and posterior to the cervical cord. This finding may reveal the decompression of the cord.

This result seems to suggest that the relative narrowing of the cervical subaranchnoid space may cause an intermittent compression and/or an intermittent hyperelongation of the spinal cord due to compression during neck movement. This mechanism may produce some clinical syndrome of whiplash injury. The relative narrowing of the subarachnoid space may result from two factors, congenital and/or acquired. 1) congenital relatve or absolute narrowing of the spinal canal to the spinal cord and congenital inequality of the growth in length between spinal cord and canal. 2) subarachnoid narrowing due to traumatic changes of the epidural soft tissue in the spinal canal; bleeding, edema, adhesion, venous congestion, hypertrophy of ligamenta flava or posteror longitudinal ligament, disc hernia, intrathecal pressure change, etc.

It seems that the individual difference of teh clinical course of whiplash injury can be explained from this mechanism.

\title{
k-20. Cerebrovascular Insufficiency at the Chronic Stage of Traumatic Cervical Syndrome
}

\author{
Minoru Aoyagi, Morio Matsunaga and Akihiko Hirayama \\ Dept. of Neurosurgery, Kurashiki Central Hospital \\ Takeshi Kurose, Juichi Ueno, Kyo Kirino and Ryosaku Ueda \\ Takahiko SAIDA \\ Dept. of Neurosurgery, Kyoto University Medical School
}

Cerebrovascular insufficiency is not infrequently observed at the chronic stage of traumatic cervical syndrome. Any type of the insufficient cerebrovascular disorders could be incident. They are conventionally divided into three types: 1) vertebral basilar insufficiency, 2) carotid basilar insufficiency, and 3) carotid insufficiency. Cervical hemodynamics still remain problematical in the cases of the traumatic cervical syndrome with these insufficiencies.

Fifty seven patients in our series of the chronic traumatic cervical syndrome with such cerebrovascular insufficiencies were studied by means of our technique of the subclavian or brachioaxillar catheterization angiography. Especially, dynamic movements of the head and neck were analyzed angiographically. It 